\title{
Social Networks Sites (SNSs) Available to Promotion of Biotechnology Summit (BS12 \& BS14) \& Int. Biotechnol. Color J.
}

\author{
Susana Lozano-Muñizi,2, José Antonio Hernández-Contreras ${ }^{1,2}$, \\ José Antonio Rosales-Barrales ${ }^{1}$ \\ ${ }^{1}$ Biotechnology Institute, Universidad del Papaloapan, Tuxtepec, Mexico \\ ${ }^{2}$ International Foundation for Biotechnology Research \& Early Stimulation in the Culture of Health, Nutrition, \\ Sport, Art, Science, Technology \& Society A.C. Nonprofit Organization, San Juan Bautista Tuxtepec, Mexico \\ Email: Susana_lozano@hotmail.com, president@bio.edu.mx
}

Received April 2014

\section{Abstract}

Social network sites (SNSs) such as Instagram, Google+, LinkedIn, Twitter, Facebook, BioWebSpin, Blogger are increasingly attracting the attention of millions users, academic and industry researchers intrigued by their affordances and reach. They can vary in the extent to which they incorporate new information and communication tools, such as mobileconnectivity, blogging, and photo/video-sharing. SNSs have the potential to increase the reach and efficiency of essential Open Access Publishing Journals, such as researches and communications. Objective: The article showed the different kinds of SNSs available in promotion of the aim of the International Foundation for Biotechnology Research \& Early Stimulation in the Culture of Health, Nutrition, Sport, Art, Science, Technology and Society civil association, a nonprofit organization (IFFBRAESITCOHNSASTAS A.C.) biotechnology summit 2012 \& 2014 (BS12 \& BS14) as the publication of International Biotechnology Color Journal (Int. Biotechnol. Color J.). Methods: We performed a systematic review and use of the social networks available in this area of the world as Instagram, Google+, Twitter, LinkedIn, Facebook (Fb), BioWebSpin\& Blogger. Results: A total of 7 SNSs were used to promote BS12, BS14 and the Int. Biotechnol. Color J. publication. The first three SNSs did not provide statistics or graphical analyses of the behavior of the network. The system provided the number of followers, following, post, and the comments and the option to share or how qualify the post; the last four SNSs systems offer statistical analyses of various aspects as set forth in the figures. Conclusion: The number of organization that uses SNSs has been steadily increasing in the past 9 years. SNSs available and trending in this part of the world can be useful to promotion of events as BS12, BS14 and the Int. Biotechnol. Color J. publication.

\section{Keywords}

Social Network Sites, International Research Journals, Biotechnology Journals, Open Access Publishing Journals, Non Profit Scholar Organizations 


\section{Introduction}

Social network sites (SNSs) are increasingly attracting the attention of academic and industry researchers intrigued by their affordances and reach [1]. International Foundation for Biotechnology Research \& Early Stimulation in the Culture of Health, Nutrition, Sport, Art, Science, Technology and Society, is a Civil Association (IFFBRAESITCOHNSASTASA.C.) currently under the development as a Web Based Business Management 1.0 http://www.bio.edu.mx [2]. It is a nonprofit organization founded in 2009 at Huajuapan de León, Oaxaca, Mexico. Actually, the aims of the foundation are the official trimonthly publication "International Biotechnology Color Journal” (Int. Biotechnol. Color J.) since October 2011. It organizes the Biotechnology Summit every two years, in 2012 (BS12) which was the 1st General Meeting [3], and actually it organizes the Biotechnology Summit (BS14) in next October from 8 to 10, 2014 at Universidad del Mar campus Huatulco, in Huatulco Oaxaca Mexico. SNSs are used to support promotion of the event and as invitation to publish the Int. Biotechnol. Color J. The journal is a nonprofit publication with genuine scientific and academic interest [4]. ISSN2226-0404 was obtained from The ISSN International Center at 45, Rue de Turbigo, 75003 in Paris France. This journal is now indexing at Universidad Nacional Autonóma de Mexico (UNAM) in Regional Information System for Online Scientific Journals from Latin America, the Caribbean, Spain and Portugal (Latindex) with the number: 20,842 [5]. The indexing at EBSCO is actually in progress. The webpage of the journal is: (http://www.ibcj.org.mx) hosted by Centro de Investigación Científica de Yucatán, A.C. (CICY) \& Produced by UNAM. Int. Biotechnol. Color J. publish original papers and articles on all aspects of biotechnology. Its aim is to provide an excellent resource for the publication of peer-reviewed research papers and articles on a wide range of the biotechnology application organized as rainbow colors with proven or likely implications for the past, current and future practice of biotechnology. The foundation is now listed in the Yearbook of International Organizations with reference number: J8276, at Union of International Associations. The foundation has the number 12999 in National Record of Science and Technology Institutes and Companies (RENIECYT) from National Council of Science and Technology (CONACYT).

\section{Objective}

The objective of this document is showing the different kind of SNSs available in promotion of the aim of IFFBRAESITCOHNSASTAS A.C. as BS12 \& BS14 organization \&Int. Biotechnol. Color J. publication.

\section{Methods}

We performed a systematic review and use of the social networks available in this area of the World as Instagram, Google+, Twitter, LinkedIn, Facebook, BioWebSpin \& Blogger to try to promote BS12, BS14 and IBCJ publication. A total of 7 SNSs was use to promote BS12, BS14 and Int. Biotechnol. Color J. publication.

\section{Results}

A total of 7 SNSswasusetopromote BS12, BS14 andInt. Biotechnol. Color J. publication. As: Instagram, Google+, Twitter, LinkedIn, Facebook, BioWebSpin, and Blogger. The first three SNSs (Instagram, Google+ \& Twitter) do not provide statistical or graphical analysis of the behavior of the network, the system provided the number of followers, following, post, and the comments and the option to share or how qualify the post; in the last four SNSs (LinkedIn, Facebook, BioWebSpin \& Blogger) systems offers statistical analysis of various aspects as set forth in the Figure 1.

\section{Social Networks}

At Instagram joined since 2014 as: “@biotechnologyresearch”, 13 posts, 32 followers, 48 following [6]. At Google+ as: "international foundation for biotechnology research" [7] are 35 followers and 17.267 views. If you want to measure activity within your Google+ profile you can do it by accessing Social Statistics (http://socialstatistics.com/), you know the trend per hour day week. Another option is Allmyplus (http://www.allmyplus.com), other is Simply Measured (http://simplymeasured.com). At Twitter joined since January 42010 as: “@InterFound4BioT”, 628 followers, 416 following, and 173 Tweets [8]. If you want to measure activity within your Twitter profile you can do it by accessing Tweet Reach (https://tweetreach.com/). 


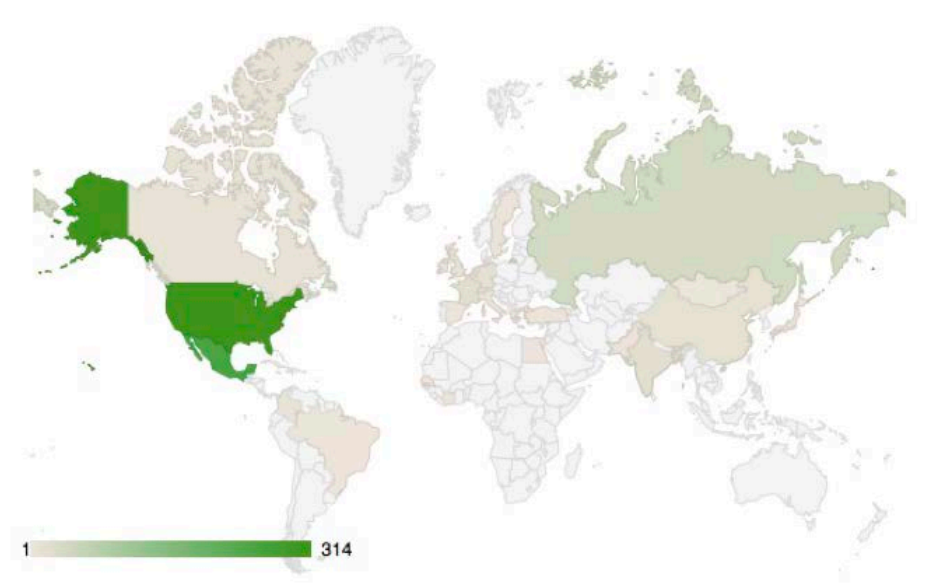

Figure 1. Statistics of the biotechnology summit 2014 (BS14) event at BioWebSpin by Visitor location.

At Linkedinprofile as: "IFfBRaESitCHNSASTaS A.C. Non Profit Org" with 500 connections [9], and as a company "IntFound 4 BioT Research \& EarlyStim in the Cult of Health, Nutr, Sport, Art, Sc, Tech \& Soc AC", with 56 followers [10]. At analitics in part of Updates, display information about: Preview, Date, Audience, Sponsored, Impressions, Clicks, Interactions, Followers, Acquired, Engagement, in updated graphics display data in a Date Range since last week until 6 months ago, Reach: Impressions \& Uniques, Engagement: Clicks, Likes, Comments, Shares, Followers, Acquired, Engagement \%. In Followers you can see the Follower demographics data as: Seniority: Senior 35.7\%, Entry 26.2\%, Owner 11.9\%, Manager 9.5\%, Training 9.5\%. At Industry: Biotechnology 22.7\%, Pharmaceuticals 9.1\%, Philanthropy 6.8\%, Nonprofit Organization Management 6.8\%, Hospital \& Health Care 6.8\%. Company Size: 10,000+ 19\%, 1 - 10 19\%, 11 - 50 19\%, 1001 - 5000 14.3\%, 51 - 200 9.5\%. Function: Research 24.4\%, Entrepreneurship 11.1\%, Education 11.1\%, Sales 6.7\%, Media and Communication 4.4\%. Employee: Non-Employee 95.9\%, Employee 4.1\%. The Follower trends display data from last week to last year. At Fb joined since April 20, 2009 as: "International foundation for Biotechnology research” [11] with 2497 Friends, Followers 32, Following 48. In biotechnology summit 2014 event [12], the guest list is: 128 going, 143 maybe and $2.2 \mathrm{~K}$ invited. The Fan pages are, ones as: "International Biotechnology Color Journal, www.ibcj.org.mx" open since November 8, 2012 with 184 likes [13] and other as: "Int Found for Biotech Research and Early Stimulation A C Nonprofit Org" [14] open since April 6, 2010, with 171 likes. Weekly page updated is sending it by electronic mail (e-mail) to the administrator. Statistical information is available about the fan page at fb as Activity \& Insights, in Activity: notification and messages, and at Insights: Overview, Likes, Reach, Visits, Posts and People. OVERVIEW, shows data in a week about: page likes, post reach and Engagement, analysis of your 5 most recent post as: date published, post, type, targeting, reach, engagement $\&$ an option to promote with boost post, the charge to promote event is 60 dollars per day. Pages to Watch to compare the performance of your Page and posts with similar Pages on Facebook \& Suggested pages to watch. LIKES: total page likes as today with information since 1 week or you can change as long as 8 months ago, for example since 8/6/2013 to 4/21/2014; and LIKES also show: total Page Likes as of Today, Net Likes: What Changed Likes-unlikes $=$ net likes \& Where Your Page Likes Came From: The number of times your Page was liked, broken down by where it happened as: Page Suggestions, On Your Page, Search, Application Programming Interface (API), Uncategorized Desktop. REACH, Post Reach: The number of people who saw your posts: Organic \& Paid; Likes, Comments, and Shares; Hide, Report as Spam, and Unlikes. Total Reach. VISITS, Page and Tab Visits as: Timeline, Photos Tab, insights, Info Tab; Other Page Activity, External Referrers. POST, When Your Fans Are Online, Post Types, Top Posts from Pages to Watch, All Posts Published. PEOPLE, Your Fans, People Reached, People Engaged in statistics data about \% Women and \% of Men kind of fans \& the country and cities of the fans and the kind of Language they speak. At BioWebSpin joined since 2013 as: "INTERNATIONAL FOUNDATION" since 2013 [15] with Key Opinion Ladders (KOL) to show other SNSs as LinkedIn, Twitter, Fb and official website with 28 Networkers, the event Biotechnology summit 2014 (BS14) has been seen 1143 times [16] you can see Last Visits, Top Visitors \& Statistics. At statistics you can see visitor 
location and number view: from Russian Federation 40, Mongolia 1, China 13, Japan 3, India 18, Pakistan 3 , Egypt 1, Turkey 2, Greece 1, Italy 2, France 18, Germany 12, Spain 2, Cote D'ivoire 2, Sengal 1, United Kingdom 4, Ireland 3, Sweden 4, Canada 9, United State 314, Mexico 249, Colombia 5, Brazil 1 (Figure 1). How your visitor come on your event; How does persons goes on your profil, Direct Access, Other Biowebspin Page, Search Engine, Social Network, Other websites, \& How often people viewed your event by number of visits.

If you want to promote the event at BioWebSpin will be 5\% for Biowebspin and 5\% for PayPaL, 10\% commission in all. The system of the event let you see the statistic information.

In blogger as: "Intbiotechnology Color Journal" [17] are 18 post and 4649 views with no followers since 8/8/10, and as: "intfound 4 biotechresearch" [18] with 8 post and 2681 views with no followers since 22/7/10.

\section{Conclusion}

The number of organization that uses SNSs has been steadily increasing in the past 9 years. SNSs available and trending in this part of the world can be useful to promotion of events as BS12, BS14 and the Int. Biotechnol. Color J. publication.

\section{References}

[1] Boyd, D.M. and Ellison, N.B. (2014) Social Network Sites: Definition, History, and Scholarship. http://www.danah.org/papers/JCMCIntro.pdf

[2] Web 1.0 (2014) http://es.wikipedia.org/wiki/Web 1.0

[3] Muñiz, S.L. (2014) Report of the Event, Biotechnology Summit 2012. International Biotechnology Color Journal, 2, 11-16. http://www.cicy.mx/Sitios/Journal/pdf/2012/October-Abastract/ibcj_2i3y2012octoberFhrp11-16.pdf

[4] Rodríguez-Sotres, R. and Plasencia, J. (2014) Internationa Biotechnology Color Journal Is a Non-Profit Publication with Genuine Scientific and Academic Interest. International Biotechnology Color Journal, 3, 6-11. http://www.cicy.mx/Sitios/Journal/pdf/2013/June/ibcj_v3i2p6-11y2013.pdf

[5] (2014) http://www.latindex.unam.mx/buscador/ficRev.html?folio=20842

[6] (2014) http://instagram.com/biotechnologyresearch

[7] (2014) https://plus.google.com/u/0/+susanalozanomuñiz/posts

[8] (2014) https://twitter.com/InterFound4BioT

[9] (2014) http://www.linkedin.com/profile/view?id=44887922\&trk=nav responsive tab profile

[10] (2014) http://www.linkedin.com/company/intfound-4-biot-research-\&-earlystim-in-the-cult-of-health-nut-art-sc-tech-\&-soc-ac -a-non-profit?trk=prof-following-company-logo

[11] (2014) https://www.facebook.com/Holaqtalholahola

[12] (2014) https://www.facebook.com/events/361328613935320/

[13] (2014) https://www.facebook.com/pages/International-Biotechnology-Color-Journal-wwwibcjorgmx/104876259551516?ref= $\underline{\mathrm{hl}}$

[14] (2014)

https://www.facebook.com/pages/Int-Found-for-Biotech-Research-and-Eearly-Stimulation-A-C-Non-profit-Org/11157 4052203338?ref=hl

[15] (2014) http://www.biowebspin.com/members/susana-lozano/

[16] (2014) http://www.biowebspin.com/events-v2/biotechnology-summit-2014/

[17] (2014) http://intbiotechnologycolorjournal.blogspot.mx

[18] (2014) http://intfound4biotechresearch.blogspot.mx 